\title{
ON THE DIFFERENTIABILITY OF ARBITRARY REAL-VALUED SET FUNCTIONS. I $\left(^{1}\right)$
}

\author{
BY
}

HARVEL WRIGHT AND W. S. SNYDER

Introduction. Lebesgue studied the differentiability of additive functions of subsets of Euclidean $n$-space. He proved [5, p. 419] that the derivative of a completely additive function of bounded variation defined on a class of intervals exists and is finite almost everywhere. He also proved [5, p. 399] that the derivative of a completely additive and absolutely continuous function defined on the class of all measurable sets exists and is finite almost everywhere, and, furthermore, the function is the indefinite integral of a function which is equal to the derivative almost everywhere. A number of later publications on the subject (see, for instance, Banach [1], R. C. Young [13], Saks [11, p. 152], Morse [8], Ward [12], de Possel [10], and Hartnett and Kruse [4]) give conditions which are sufficient for the existence of a derivative under more general conditions, i.e., for functions that are not necessarily additive, or for functions of subsets of abstract spaces. However, to the authors' knowledge, conditions have never been given which are both necessary and sufficient for the existence almost everywhere of a unique, finite derivative. It is the purpose of this paper to give such conditions. Also, necessary and sufficient conditions are given for each of the upper and lower derivates of the function to be finite almost everywhere and to be bounded almost everywhere on a measurable set of finite measure.

This investigation will be restricted to Euclidean $n$-space $R_{n}$ and to Lebesgue measure. The functions considered will be arbitrary, real and finite valued set functions. The family $\mathscr{F}$ of sets on which the functions are defined will be quite general and will not be required to be additive. The family $\mathscr{F}$ will be a collection of sets with the property that if $T$ is in $\mathscr{F}$, then $T$ is measurable and the measure of $T$ is equal to the measure of the closure of $T$. This last condition is imposed in order that the Vitali covering theorem will be available for subfamilies of $\mathscr{F}$.

Definitions and basic concepts. If $T$ is a subset of $R_{n}$, the outer measure or exterior measure of $T$ will be denoted by $|T|_{e}$ and, if $T$ is known to be measurable, the Lebesgue measure of $T$ will be denoted $|T|$.

Presented in part to the Society, January 24, 1969 under the title On the derivatives of arbitrary real valued set functions; received by the editors January 10, 1969.

( $\left.{ }^{1}\right)$ Research sponsored by the U.S. Atomic Energy Commission under contract with Union Carbide Corporation.

Copyright (C) 1969, American Mathematical Society 
DEFINITION 1. The parameter of regularity of a bounded measurable set $T$, denoted $r(T)$, is defined as the least upper bound (l.u.b.) of the set of numbers $|T| /|J|$ where $J$ denotes a cube containing $T$.

Definition 2. A sequence $\left\{T_{i}\right\}$ of sets is said to close down on the point $x$ if $x \in T_{i}$ for each $i$ and if $\lim _{i \rightarrow \infty}\left[\operatorname{diam}\left(T_{i}\right)\right]=0$, where diam $\left(T_{i}\right)$ denotes the diameter of the set $T_{i}$.

Definition 3. Let $\alpha$ represent a real number such that $0<\alpha<1$. A sequence of sets is said to be a regular $\alpha$ sequence if each of the sets in the sequence has parameter of regularity greater than $\alpha$.

Definition 4. Let $\alpha$ be a real number such that $0<\alpha<1$, let $x$ be a point in $R_{n}$, and suppose that $\mathscr{F}$ contains at least one regular $\alpha$ sequence of sets that closes down on the point $x$. Define the upper $(\mathscr{F}, \alpha)$ derivate, $D^{\circ}(\mathscr{F}, f, \alpha, x)$, of $f$ at the point $x$ to be

$$
D^{0}(\mathscr{F}, f, \alpha, x)=\lim \sup f(T) /|T|=\lim _{\Delta \rightarrow 0}[\text { l.u.b. } f(T) /|T|]
$$

and the lower $(\mathscr{F}, \alpha)$ derivate, $D_{0}(\mathscr{F}, f, \alpha, x)$, of $f$ at $x$ to be

$$
\left.D_{0}(\mathscr{F}, f, \alpha, x)=\lim \inf f(T) /|T|=\lim _{\Delta \rightarrow 0} \text { [g.l.b. } f(T) /|T|\right]
$$

where the limit superior (lim sup) and limit inferior (lim inf) are taken as diam $(T)$ $\rightarrow 0$ for sets $T$ such that $x \in T, T \in \mathscr{F}$, and $r(T)>\alpha$, and the least upper bound (l.u.b.) and greatest lower bound (g.l.b.) are taken over sets $T$ such that $x \in T$, $T \in \mathscr{F}, r(T)>\alpha$, and $\operatorname{diam}(T)<\Delta$.

The l.u.b. and g.l.b. are monotone functions of $\Delta$. Therefore, the limit always exists but may have the value $+\infty$ or $-\infty$. In order to simplify notation, reference to the family $\mathscr{F}$ will be deleted from the symbol. Thus $D^{0}(\mathscr{F}, f, \alpha, x)$ and $D_{0}(\mathscr{F}, f, \alpha, x)$ will be denoted simply $D^{0}(f, \alpha, x)$ and $D_{0}(f, \alpha, x)$, respectively. These derivates are defined only at points $x$ where there exists a regular $\alpha$ sequence of sets from the family $\mathscr{F}$ that closes down on $x$. The set of all such points constitutes the domain of definition of the derivates and will be denoted by $S_{\alpha}$.

REMARK 1. $D^{0}(f, \alpha, x)$ and $D_{0}(f, \alpha, x)$ are, respectively, the least upper bound and greatest lower bound of numbers $L$ for which there exists a regular $\alpha$ sequence $\left\{T_{i}\right\}$ closing down on $x$ such that $\lim _{i \rightarrow \infty} f\left(T_{i}\right) /\left|T_{i}\right|=L$. Furthermore, there is a sequence $\left\{T_{i}\right\}$ for which $\lim _{i \rightarrow \infty} f\left(T_{i}\right) /\left|T_{i}\right|=D^{0}(f, \alpha, x)$ and a sequence $\left\{T_{j}\right\}$ for which $\lim _{j \rightarrow \infty} f\left(T_{j}\right) /\left|T_{j}\right|=D_{0}(f, \alpha, x)$. The existence of such sequences follows from the definitions and by use of the diagonal process.

If the upper and lower $(\mathscr{F}, \alpha)$ derivates are equal, the function $f$ is said to be $(\mathscr{F}, \alpha)$ differentiable at $x$ with $(\mathscr{F}, \alpha)$ derivative, denoted $D(f, \alpha, x)$, equal to the common value of the derivates, which may be $\infty$ or $-\infty$.

REMARK 2. In view of the monotoneity of each of the l.u.b. and g.l.b. as a function of $\Delta$ in Definition 4 , the derivates can be written

$$
D^{0}(f, \alpha, x)=\underset{\Delta>0}{\text { g.l.b. }}[\text { l.u.b. } f(T) /|T|] \text { and } D_{0}(f, \alpha, x)=\underset{\Delta>0}{\text { l.u.b. [g.l.b. } f(T) /|T|]}
$$


where the 1.u.b. and g.l.b. in the brackets are taken over sets $T$ such that $x \in T$, $T \in \mathscr{F}, r(T)>\alpha$, and $\operatorname{diam}(T)<\Delta$.

Some of the properties of the $(\mathscr{F}, \alpha)$ derivates which are immediate consequences of the definitions and of properties of limit superior, least upper bound, etc., of real numbers (see, for instance, McShane [6, pp. 1-51]) are listed in Theorem 1. Proofs will be omitted.

THEOREM 1. Let $f$ and $g$ be real valued functions defined and finite on sets in the family $\mathscr{F}$. Then at any point $x$ in the domain $S_{\alpha}$ of definition of the $(\mathscr{F}, \alpha)$ derivates the following conditions hold:

(a) $D_{0}(f, \alpha, x)=-D^{0}(-f, \alpha, x)$.

(b) $D^{0}(a f, \alpha, x)=a D^{0}(f, \alpha, x)$ for any positive number $a$.

(c) $D^{0}(a f, \alpha, x)=a D_{0}(f, \alpha, x)$ for any negative number $a$.

(d) $D^{0}(f+g, \alpha, x) \leqq D^{0}(f, \alpha, x)+D^{0}(g, \alpha, x)$ provided the sum on the right is defined.

(e) $D_{0}(f+g, \alpha, x) \geqq D_{0}(f, \alpha, x)+D_{0}(g, \alpha, x)$ provided the sum on the right is defined.

(f) If $\beta$ is a real number with $0<\beta<\alpha$, then $D^{0}(f, \beta, x) \geqq D^{0}(f, \alpha, x)$ and $D_{0}(f, \beta, x)$ $\leqq D_{0}(f, \alpha, x)$.

(g) $D^{0}(f, \alpha, x)=D^{0}\left(f^{+}, \alpha, x\right)-D_{0}\left(f^{-}, \alpha, x\right)$ where $f^{+}(T)=\max [f(T), 0]$ and $f^{-}(T)=\max [-f(T), 0]$ denote the positive and negative parts of $f$.

Furthermore, at most one of $D^{0}\left(f^{+}, \alpha, x\right)$ and $D_{0}\left(f^{-}, \alpha, x\right)$ can be different from 0 .

(h) $D_{0}(f, \alpha, x)=D_{0}\left(f^{+}, \alpha, x\right)-D^{\circ}\left(f^{-}, \alpha, x\right)$ and at most one of $D_{0}\left(f^{+}, \alpha, x\right)$ and $D^{0}\left(f^{-}, \alpha, x\right)$ can be different from 0 .

Definition 5. Suppose $0<\beta<1$ and $x \in S_{\alpha}$ for every $\alpha$ such that $0<\alpha<\beta$. Then the $(\mathscr{F}, \alpha)$ derivates are defined at $x$ for each value of $\alpha$ and, in view of Theorem $1(\mathrm{f})$, are monotone functions of $\alpha$. Define the upper $(\mathscr{F}, 0)$ derivate, $D^{\circ}(f, x)$, of $f$ at the point $x$ to be

$$
D^{0}(f, x)=\lim _{\alpha \rightarrow 0+} D^{0}(f, \alpha, x)=\text { l.u.b. } D^{0}(f, \alpha, x)
$$

and the lower $(\mathscr{F}, 0)$ derivate, $D_{0}(f, x)$, of $f$ at the point $x$ to be

$$
D_{0}(f, x)=\lim _{\alpha \rightarrow 0+} D_{0}(f, \alpha, x)=\text { g.l.b. } D_{0}(f, \alpha, x)
$$

where the l.u.b. and g.l.b. are taken over all $\alpha$ such that $0<\alpha<\beta$.

The definition of the $(\mathscr{F}, \alpha)$ derivates at the point $x$ can be generalized somewhat by not requiring that the point $x$ be contained in the set $T$ but just requiring that $r(T \cup\{x\})>\alpha$ and diam $(T \cup\{x\}) \rightarrow 0$. The symbol $\{x\}$ is used here to denote the set whose only element is the point $x$.

Definition 6. Let $S_{\alpha}^{*}$ denote the set of all points $x$ for which there exists a sequence $T_{i}$ of sets from $\mathscr{F}$ such that $r(T \cup\{x\})>\alpha$ and $\lim _{i \rightarrow \infty}\left[\operatorname{diam}\left(T_{i} \cup\{x\}\right)\right]=0$. 
Define the upper star derivate, $D^{0 *}(f, \alpha, x)$, to be

$$
D^{0 *}(f, \alpha, x)=\lim \sup f(T) /|T|=\lim _{\Delta \rightarrow 0}[\text { l.u.b. } f(T) /|T|]
$$

and the lower star derivate, $D_{0}^{*}(f, \alpha, x)$, to be

$$
\left.D_{0}^{*}(f, \alpha, x)=\lim \inf f(T) /|T|=\lim _{\Delta \rightarrow 0} \text { [g.l.b. } f(T) /|T|\right],
$$

where the lim sup and lim inf are taken as $\operatorname{diam}(T \cup\{x\}) \rightarrow 0$ for sets $T \in \mathscr{F}$ such that $r(T \cup\{x\})>\alpha$ and where the l.u.b. and g.l.b. are taken over sets $T \in \mathscr{F}$ such that $r(T \cup\{x\})>\alpha$ and $\operatorname{diam}(T \cup\{x)\}<\Delta$.

It is seen that the set $S_{\alpha}^{*}$ is the domain of definition of $D^{0 *}(f, \alpha, x)$. Note that $S_{\alpha} \subset S_{\alpha}^{*}$ and also that at any point $x \in S_{\alpha}$ the inequalities $D^{0}(f, \alpha, x) \leqq D^{0 *}(f, \alpha, x)$ and $D_{0}(f, \alpha, x) \geqq D_{0}^{*}(f, \alpha, x)$ are satisfied. It will be shown in Theorem 4 that $\left|S_{\alpha}^{*}-S_{\alpha}\right|=0$ and, furthermore, $D^{0}(f, \alpha, x)=D^{0 *}(f, \alpha, x)$ almost everywhere on $S_{\alpha}$.

REMARK 3. Note that Theorem 1 remains valid if $D^{\circ}(f, \alpha, x)$ and $D_{0}(f, \alpha, x)$ are replaced by $D^{0 *}(f, \alpha, x)$ and $D_{0}^{*}(f, \alpha, x)$, respectively, throughout the theorem.

The proofs of the following theorems make extensive use of the Vitali covering theorem, a proof of which can be found in several places in the literature, (see, for instance, Saks [11, p. 109]) and, therefore, will not be included here. However, the theorem will be stated for convenience in a slightly more general form than usual. An argument similar to that given by Saks [11, p. 111] shows that the theorem is valid in this form.

Definition 7. A family $S$ of sets is said to cover a set $E$ in the sense of Vitali if there exists a subset $A$ of $E$ such that $|E-A|=0$ and $x \in A$ implies that for some $\alpha>0$ there exists a regular $\alpha$ sequence of sets from $S$ that closes down on $x$.

The following theorem is known as the Vitali Covering Theorem:

THEOREM 2. Let $E$ be a subset of Euclidean $n$-space $R_{n}$ and let $S$ be a family of subsets $T$ of $R_{n}$ such that $|T|=|\bar{T}|$, where $\bar{T}$ denotes the closure of $T$, that covers the set $E$ in the sense of Vitali. Then there exists in $S$ a finite or enumerable sequence $\left\{T_{n}\right\}$ of sets, no two of which have points in common, such that $\left|E-\bigcup_{n} T_{n}\right|=0$.

The Vitali covering theorem is often stated in the following form [2, p. 170]:

COROLlaRY 1. The hypotheses of Theorem 2 imply that for an arbitrary $\varepsilon>0$, there exists a finite collection $\left\{T_{i}\right\}_{i=1}^{n}$ of sets from $S$, no two of which have points in common, such that $\left|E-\bigcup_{i=1}^{n} T_{i}\right|_{e}<\varepsilon$.

The next result follows directly from the definition but will be stated for future use. Proofs will be omitted. In the interest of brevity several conditions will be included in one lemma.

LEMMA 1. Consider the unprimed conditions (1) $D^{0}(f, \alpha, x)>a$, (2) $D^{0}(f, \alpha, x)<a$, (3) $D^{\circ}(f, \alpha, x) \geqq a$, (4) $D^{\circ}(f, \alpha, x) \leqq a$, (5) $D^{\circ}(f, \alpha, x)=+\infty$, (6) $D^{\circ}(f, \alpha, x)=-\infty$, and the primed conditions $\left(1^{\prime}\right) f(T) /|T|>a,\left(2^{\prime}\right) f(T) /|T|<a,\left(3^{\prime}\right) f(T) /|T|>a-1 / n$, 
(4') $f(T) /|T|<a+1 / n,\left(5^{\prime}\right) f(T) /|T|>n$, and $\left(6^{\prime}\right) f(T) /|T|<-n$. If a is real number, $\alpha$ is a real number such that $0<\alpha<1, i$ is an integer such that $1 \leqq i \leqq 6$, and $D^{\circ}(f, \alpha, x)$ satisfies condition (i) at almost all points of a set $E$, then for any positive integer $n$ and any positive number $\Delta$, the collection of all sets $T \in \mathscr{F}$ such that $r(T)>\alpha$, $\operatorname{diam}(T)<\Delta$ and condition ( $\left.\mathrm{i}^{\prime}\right)$ is satisfied, covers $E$ in the sense of Vitali.

It is noted that Lemma 1 remains valid if $D^{0}(f, \alpha, x)$ is replaced by $D_{0}(f, \alpha, x)$, $D^{0 *}(f, \alpha, x)$, or $D_{0}^{*}(f, \alpha, x)$ throughout.

Script letters $\mathscr{E}, \mathscr{H}$, etc., will be used to denote finite collections of mutually disjoint sets from the family $\mathscr{F}$. A dot $\left(^{(}\right)$placed over a script letter will denote the point set obtained by the union of all sets in the collection, e.g., $\mathscr{E}=\bigcup_{T \in \mathscr{E}} T$. If a function $f$ is defined for each set $T \in \mathscr{E}$, then, with two exceptions, $f(\mathscr{E})$ will denote the sum of the values of the function on sets from $\mathscr{E}$, i.e., $f(\mathscr{E})=\sum_{T \in \mathscr{E}} f(T)$. The two exceptions are diam $(\mathscr{E})$ and $r(\mathscr{E})$. The symbol diam $(\mathscr{E})$ will denote the maximum of diam $(T)$ for $T \in \mathscr{E}$ and the symbol $r(\mathscr{E})$ will denote the minimum of $r(T)$ for $T \in \mathscr{E}$. Unless otherwise noted, the symbol $\mathscr{F}_{\alpha}$ will denote the family defined as $\mathscr{F}_{\alpha}=\{T \mid T \in \mathscr{F}$ and $r(T)>\alpha\}$. The symbol $\nabla$ will be used to denote the symmetric difference of two sets, i.e., for any two sets $A$ and $B, A \nabla B=(A-B) \cup(B-A)$.

Measurability of the $(\mathscr{F}, \alpha)$ derivates. Measurability of the derivates plays a vital role in this development. It will be shown that the star derivates are Borel measurable and that the $(\mathscr{F}, \alpha)$ derivates are equal to the $(\mathscr{F}, \alpha)$ star derivates almost everywhere.

THEOREM 3. If $a$ is a real number then $\left\{x \mid D^{0 *}(f, \alpha, x)>a\right\}$ is a Borel set.

Proof. It follows from the definition of $D^{0 *}(f, \alpha, x)$ that $\left\{x \mid D^{0 *}(f, \alpha, x)>a\right\}$ $=\bigcup_{n=1}^{\infty} \bigcap_{m=1}^{\infty} E_{n, m}$ where $E_{n, m}=\{y \mid$ there is a set $T \in \mathscr{F}$ such that $\operatorname{diam}(T)<1 / m$, $r(T \cup\{y\})>\alpha$, and $f(T) /|T|>a+1 / n\}$. It suffices to show that for each pair of values $m$ and $n$, the set $E_{n, m}$ is an open set. To see this, let each of $n_{0}$ and $m_{0}$ be a positive integer and let $y_{0}$ be an arbitrary point in $E_{n_{0}, m_{0}}$. Then there exists a set $T_{0} \in \mathscr{F}$ such that diam $\left(T_{0}\right)<1 / m_{0}, f\left(T_{0}\right) /\left|T_{0}\right|>a+1 / n_{0}$ and $r\left(T_{0} \cup\left\{y_{0}\right\}\right)>\alpha$. From the definition of $r\left(T_{0} \cup\left\{y_{0}\right\}\right)$, there exists a cube $J$ such that $\left(T_{0} \cup\left\{y_{0}\right\}\right) \subset J$ and $\left|T_{0} \cup\left\{y_{0}\right\}\right| /|J|>\alpha$. But since this last is a strict inequality, there exists another cube $J^{\prime}$ containing $J$ in its interior and satisfying $\left|T_{0} \cup\left\{y_{0}\right\}\right| /\left|J^{\prime}\right|>\alpha$. Now any point in $J^{\prime}$ is in $E_{n_{0}, m_{0}}$ and since $y_{0}$ is in the interior of $J^{\prime}$, there is some neighborhood of $y_{0}$ which is in $J^{\prime}$. But this neighborhood of $y_{0}$ is a subset of $E_{n_{0}, m_{0}}$ and the theorem is proved.

The relationship between the $(\mathscr{F}, \alpha)$ derivates and the $(\mathscr{F}, \alpha)$ star derivates is given in the following theorem.

THEOREM 4. If $0<\alpha<1$, then $\left|S_{\alpha}^{*}-S_{\alpha}\right|=0$. Furthermore, $D^{0}(f, \alpha, x)=D^{0 *}(f, \alpha, x)$ and $D_{0}(f, \alpha, x)=D_{0}^{*}(f, \alpha, x)$ almost everywhere on $S_{\alpha}$.

Proof. Assume $\left|S_{\alpha}^{*}-S_{\alpha}\right|_{e}>0$. Then there exists a subset $A$ of $S_{\alpha}^{*}-S_{\alpha}$ such that $0<|A|_{e}<+\infty$. Let $n$ be a positive integer. By Lemma 1 , the collection $\mathscr{F}^{n}=$ 
$\{T \mid T \in \mathscr{F}, x \in A, r(T \cup\{x\})>\alpha$, and $\operatorname{diam}(T \cup\{x\})<1 / n\}$ covers $A$ in the sense of Vitali. Then by the Vitali covering theorem there exists a sequence $\left\{T_{i}^{n}\right\}_{i=1}^{\infty}$ of sets from $\mathscr{F}^{n}$ such that $\left|A-\bigcup_{i} T_{i}^{n}\right|=0$. Let $A^{n}=\bigcup_{i} T_{i}^{n}$. A set $A^{n}$ is thus determined for each positive integer $n$. Now $A=\left(A-A^{n}\right) \cup\left(A \cap A^{n}\right)$ so that $|A|_{e} \leqq\left|A-A^{n}\right|_{e}$ $+\left|A \cap A^{n}\right|_{e}$ and since $\left|A-A^{n}\right|=0,\left|A \cap A^{n}\right|_{e} \geqq|A|_{e}$. Thus, since $|A|_{e}<+\infty$, it follows that $\left|\lim \sup _{n \rightarrow \infty}\left(A \cap A^{n}\right)\right|_{e} \geqq|A|_{e}>0$ which implies that there is a point $x_{0}$ in $\lim \sup _{n \rightarrow \infty}\left(A \cap A^{n}\right)$. But such a point must be in $A \cap A_{n}$ for infinitely many values of $n$. Consequently, $x_{0} \in S_{\alpha}$ which is a contradiction since $x_{0} \in A$ and $A \subset\left(S_{\alpha}^{*}-S_{\alpha}\right)$. Thus $\left|S_{\alpha}^{*}-S_{\alpha}\right|=0$ and the first part of the theorem is proved.

It will now be shown that $D^{0}(f, \alpha, x)=D^{0 *}(f, \alpha, x)$ almost everywhere on $S_{\alpha}$. Suppose the contrary. Then there is a set $A \subset S_{\alpha}$ and a rational number $b$ such that $0<|A|_{e}<+\infty$ and, at each point $x \in A, D^{0}(f, \alpha, x)<b<D^{0 *}(f, \alpha, x)$. Let $n$ be a positive integer. By Lemma 1 , since $D^{0 *}(f, \alpha, x)>b$ at each point $x \in A$, the collection $\mathscr{F}^{n}=\{T|T \in \mathscr{F}, r(T \cup\{x\})>\alpha, f(T) /| T \mid>b$, and $\operatorname{diam}(T \cup\{x\})<1 / n\}$ covers $A$ in the sense of Vitali. By the Vitali covering theorem there is a sequence $\left\{T_{i}^{n}\right\}_{i=1}^{\infty}$ of sets from this collection for which $\left|A-\bigcup_{i} T_{i}^{n}\right|=0$. Let $A^{n}=\bigcup_{i} T_{i}^{n}$. An argument similar to that used in the first part of this theorem shows that $\left|\lim \sup _{n \rightarrow \infty}\left(A \cap A^{n}\right)\right|_{e} \geqq|A|_{e}>0$ so that there must exist a point $x_{0}$ in

$$
\limsup _{n \rightarrow \infty}\left(A \cap A^{n}\right) \text {. }
$$

But, for such a point $x_{0}, D^{0}\left(f, \alpha, x_{0}\right) \geqq b$ which contradicts $D^{0}\left(f, \alpha, x_{0}\right)<b$. Thus the two upper derivates are equal almost everywhere on $S_{\alpha}$. A similar argument shows that $D_{0}(f, \alpha, x)=D_{0}^{*}(f, \alpha, x)$ almost everywhere on $S_{\alpha}$ and the theorem is proved.

Thus the two derivates $D^{0}(f, \alpha, x)$ and $D^{0 *}(f, \alpha, x)$ are found to be essentially the same. Since $D^{0 *}(f, \alpha, x)$ is measurable, Theorem 4 provides a different proof of the measurability of $D^{\circ}(f, \alpha, x)$ than that given by Hahn and Rosenthal [3, p. 274] and Hartnett and Kruse [4, p. 187]. However, $D^{0 *}(f, \alpha, x)$ is of special interest because it is Borel measurable. Hartnett and Kruse [4, p. 188] defined a derivate which represents a generalization of $D^{\circ}(f, \alpha, x)$ and claimed a proof of the Borel measurability of this derivate. However, a counterexample was given by Pauc [9].

The final result on measurability that will be stated follows from the measurability of $D^{\circ}(f, \alpha, x)$ and $D_{0}(f, \alpha, x)$ and the fact that the limit function of a sequence of measurable functions is a measurable function.

Corollary 2. $D^{0}(f, x)$ and $D_{0}(f, x)$ (cf. Definition 5) are measurable functions of $x$.

Finiteness of the $(\mathscr{F}, \alpha)$ derivates. This section contains theorems giving necessary and sufficient conditions that the derivates be finite almost everywhere on a measurable set $E$ of finite measure. The conditions will be given separately for the upper $(\mathscr{F}, \alpha)$ derivate and for the lower $(\mathscr{F}, \alpha)$ derivate. It follows from Theorem 
4 that these theorems remain true if the $(\mathscr{F}, \alpha)$ derivates are replaced by the $(\mathscr{F}, \alpha)$ star derivates throughout.

THEOREM 5. Let $E$ be a measurable set with $|E|<+\infty$, let $\alpha$ be a real number such that $0<\alpha<1$, and assume that $\left|E-S_{\alpha}\right|=0$. A necessary and sufficient condition that $D^{0}(f, \alpha, x)<+\infty$ almost everywhere on $E$ is that for any $\Delta>0$, there exist numbers $a$ and $\xi>0$ such that for any $\mathscr{E}$ with $r(\mathscr{E})>\alpha$ and $\operatorname{diam}(\mathscr{E})<\xi$, the subcollection $\mathscr{E}^{\prime}$ of $\mathscr{E}$ consisting of those sets $T$ of $\mathscr{E}$ for which $f(T)>a|T|$ has $\left|\mathscr{E}^{\prime} \cap E\right|<\Delta$.

Proof. To show sufficiency, assume that the condition holds and deny the conclusion, i.e., suppose that $D^{\circ}(f, \alpha, x)=+\infty$ on a set $A \subset E$ with $|A|>0$. Let $\Delta=|A| / 2$ and let $a$ and $\xi$ be numbers satisfying the condition. By Lemma 1 , the collection $\mathscr{F}^{\prime}=\left\{T \mid T \in \mathscr{F}_{\alpha}\right.$, diam $(T)<\xi$ and $\left.f(T)>a|T|\right\}$ covers $A$ in the sense of Vitali. Then by Corollary 1 , there is a collection $\mathscr{E}$ of sets from $\mathscr{F}^{\prime}$ such that $|A-\mathscr{E}|<|A| / 2$. But $|A \cap \mathscr{E}|=|A|-|A-\mathscr{E}|>|A| / 2=\Delta$ and $|E \cap \mathscr{E}| \geqq|A \cap \mathscr{E}|$ which contradicts the hypothesis that the condition holds.

To show necessity, assume $D^{0}(f, \alpha, x)<+\infty$ almost everywhere on $E$. Suppose the condition fails to hold. Let $\Delta$ be a value for which there exists no pair of numbers $a$ and $\xi$ satisfying the condition. Then, for each integer $n$, there must exist a collection $\mathscr{E}_{n}$ such that $\left|E \cap \mathscr{E}_{n}\right|>\Delta$ and such that $r(T)>\alpha$, diam $(T)<1 / n$ and $f(T)>n|T|$ for each $T \in \mathscr{E}_{n}$. Since $|E|<+\infty$, it follows that $\left|E \cap \lim \sup \mathscr{E}_{n}\right| \geqq \Delta$. But at each point $x$ of lim sup $\mathscr{E}_{n}, D^{0}(f, \alpha, x)=+\infty$ which contradicts the hypotheses and the theorem is proved.

Corollary 3. A necessary and sufficient condition that $D_{0}(f, \alpha, x)>-\infty$ is obtained by replacing, in Theorem $5, D^{\circ}(f, \alpha, x)<+\infty$ by $D_{0}(f, \alpha, x)>-\infty$ and the condition $f(T)>a|T|$ by $f(T)<a|T|$.

The proof follows from Theorem 5 and the observation, stated in Theorem 1(a), that $D_{0}(f, \alpha, x)=-D^{0}(-f, \alpha, x)$.

The next theorem gives conditions that $D_{0}(f, \alpha, x)<+\infty$ almost everywhere.

THEOREM 6. Let $E$ be measurable with $|E|<+\infty$, let $\alpha$ be a real number such that $0<\alpha<1$, and assume $\left|E-S_{\alpha}\right|=0$. A necessary and sufficient condition that $D_{0}(f, \alpha, x)$ $<+\infty$ almost everywhere on $E$ is that for any $\varepsilon>0$ there is a number $a_{\varepsilon}$ and a sequence $\left\{\mathscr{E}_{n}\right\}_{n=1}^{\infty}$ such that, for each positive integer $n,\left|E-\mathscr{E}_{n}\right|<\varepsilon+1 / n$, and $T \in \mathscr{E}_{n}$ implies $r(T)>\alpha, \operatorname{diam}(T)<1 / n$ and $f(T)<a_{\varepsilon}|T|$.

Proof. To show sufficiency assume the condition holds. For an arbitrary $\varepsilon>0$, let $a_{\varepsilon}$ and $\left\{\mathscr{E}_{n}\right\}_{n=1}^{\infty}$ satisfy the condition. Now $|E|=\left|E \cap \mathscr{E}_{n}\right|+\left|E-\mathscr{E}_{n}\right|$ and hence $\left|E \cap \mathscr{E}_{n}\right|>|E|-\varepsilon-1 / n$. Let $B=\lim \sup \left(E \cap \mathscr{E}_{n}\right)$. Since $|E|<+\infty$,

$$
|B| \geqq \lim \sup \left|E \cap \dot{\mathscr{E}}_{n}\right| \geqq|E|-\varepsilon .
$$

But $D_{0}\left(f, \alpha, x_{0}\right) \leqq a_{\varepsilon}$ for any point $x_{0} \in B$. Thus for an arbitrary $\varepsilon>0, D_{0}(f, \alpha, x) \leqq a_{\varepsilon}$ on all of $E$ except at most a set of measure $<\varepsilon$ and, therefore, $D_{0}(f, \alpha, x)<+\infty$ almost everywhere on $E$. 
To show necessity, assume $D_{0}(f, \alpha, x)<+\infty$ almost everywhere on $E$. Let $\varepsilon>0$ be arbitrary. Let $E_{n}=\left\{x \mid x \in E\right.$ and $\left.D_{0}(f, \alpha, x)<n\right\}$. Then $\lim _{n}\left|E_{n}\right|=|E|$ by hypotheses. Let $N$ be an integer such that $\left|E-E_{N}\right|<\varepsilon$. Let $a_{\varepsilon}=N+1$. By Lemma 1, it follows that for each positive integer $n$, the collection

$$
\mathscr{F}^{n}=\left\{T \mid T \in \mathscr{F}_{\alpha} \text {, diam }(T)<1 / n \text {, and } f(T)<a_{\varepsilon}|T|\right\}
$$

covers $E_{N}$ in the sense of Vitali. By the Vitali covering theorem there is a collection $\mathscr{E}_{n} \subset \mathscr{F}^{n}$ such that $\left|E_{N}-\mathscr{E}_{n}\right|<1 / n$. Thus $\left|E-\mathscr{E}_{n}\right|=\left|E-E_{N}\right|+\left|E_{N}-\mathscr{E}_{n}\right|<\varepsilon+1 / n$ and the theorem is proved.

Corollary 4. A necessary and sufficient condition that $D^{0}(f, \alpha, x)>-\infty$ is obtained by replacing, in Theorem $6, D_{0}(f, \alpha, x)<\infty$ by $D^{0}(f, \alpha, x)>-\infty$ and the condition $f(T)<a_{\varepsilon}|T|$ by $f(T)>a_{\varepsilon}|T|$.

The proof follows from Theorem 6 and Theorem 1(a).

The above two theorems and two corollaries give necessary and sufficient conditions that the upper and lower $(\mathscr{F}, \alpha)$ derivates be finite almost everywhere on a measurable set of finite measure.

Boundedness of the $(\mathscr{F}, \alpha)$ derivates. This section contains theorems giving necessary and sufficient conditions that the upper and lower $(\mathscr{F}, \alpha)$ derivates be bounded almost everywhere on a measurable set of finite measure. It follows from Theorem 4 that these theorems are true if the $(\mathscr{F}, \alpha)$ derivates are replaced by the $(\mathscr{F}, \alpha)$ star derivates throughout.

THEOREM 7. Let $E$ be measurable with $|E|<+\infty$, let $\alpha$ be a real number with $0<\alpha<1$, let $a$ be a real number, and assume that $\left|E-S_{\alpha}\right|=0$. A necessary and sufficient condition that $D^{\circ}(f, \alpha, x) \leqq a$ almost everywhere on $E$ is that for any $\varepsilon>0$ and $\Delta>0$, there exists a number $\xi>0$ such that for any $\mathscr{E}$ satisfying $r(\mathscr{E})>\alpha$ and $\operatorname{diam}(\mathscr{E})<\xi$, the subcollection $\mathscr{E}^{\prime}$ of $\mathscr{E}$ consisting of all sets $T \in \mathscr{E}$ for which $f(T)>(a+\varepsilon)|T|$ has $\left|\mathscr{E}^{\prime} \cap E\right|<\Delta$.

To prove sufficiency, assume the condition holds. Suppose the contrary of the conclusion, i.e., suppose there is a subset $A$ of $E$ such that $|A|>0$ and such that $D^{\circ}(f, \alpha, x)>a$ for each $x \in A$. Then there is a subset $B$ of $A$ and a number $\eta>0$ such that $|B|>0$ and $D^{0}(f, \alpha, x)>a+\eta$ for each $x \in B$. A contradiction will be reached by showing that there exist positive numbers $\varepsilon$ and $\Delta$ such that for any $\xi$ there is an $\mathscr{E}$ for which the condition fails. Take $\varepsilon<\eta / 2$ and $\Delta<|B| / 2$. Let $\xi$ be an arbitrary positive number. By Lemma 1 , the collection

$$
\mathscr{F}^{\prime}=\left\{T \mid T \in \mathscr{F}_{\alpha}, \operatorname{diam}(T)<\xi \text { and } f(T)>(a+\varepsilon)|T|\right\}
$$

covers $B$ in the sense of Vitali and, therefore, by the Vitali covering theorem there is a collection $\mathscr{E}$ such that $|B-\mathscr{E}|<\Delta / 2$. But $|B \cap \mathscr{E}|=|B|-|B-\mathscr{E}|>3 \Delta / 2$, which is a contradiction of the hypothesis that a value of $\xi>0$ exists for which the condition holds. 
To show necessity of the condition, assume $D^{0}(f, \alpha, x) \leqq a$ almost everywhere on $E$. Suppose the condition fails to hold. Let $\varepsilon>0$ and $\Delta>0$ be a pair of values for which no value for $\xi$ will satisfy the condition. For each positive integer $n$, let $\xi=1 / n$ and let $\mathscr{E}_{n}$ be a collection such that $\left|\mathscr{E}_{n} \cap E\right| \geqq \Delta$ and such that $T \in \mathscr{E}_{n}$ implies $r(T)>\alpha$, diam $(T)<1 / n$ and $f(T)>(a+\varepsilon)|T|$. Since $|E|<+\infty$,

$$
\left|E \cap \lim \sup \mathscr{E}_{n}\right| \geqq \Delta \text {. }
$$

But $D^{\circ}(f, \alpha, x) \geqq a+\varepsilon$ for each point $x \in \lim \sup \left(E \cap \mathscr{E}_{n}\right)$ which contradicts the assumption that $D^{0}(f, \alpha, x) \leqq a$ almost everywhere on $E$.

Corollary 5. A necessary and sufficient condition that $D_{0}(f, \alpha, x) \geqq a$ almost everywhere on $E$ can be obtained from Theorem 7 by replacing $D^{\circ}(f, \alpha, x) \leqq a$ by $D_{0}(f, \alpha, x) \geqq a$ and the condition $f(T)>(a+\varepsilon)|T|$ by $f(T)<(a-\varepsilon)|T|$.

The proof follows from Theorem 7 and Theorem 1(a).

THEOREM 8. Let $E$ be measurable with $|E|<+\infty$, let $\alpha$ be a real number such that $0<\alpha<1$, let a be a real number and assume $\left|E-S_{\alpha}\right|=0$. A necessary and sufficient condition that $D_{0}(f, \alpha, x) \leqq a$ almost everywhere on $E$ is that if $\varepsilon$ is an arbitrary positive number then for each integer $n>0$ there exists a collection $\mathscr{E}$ such that $\left|E-\mathscr{E}_{n}\right|<1 / n, r\left(\mathscr{E}_{n}\right)>\alpha$, diam $\left(\mathscr{E}_{n}\right)<1 / n$ and $T \in \mathscr{E}_{n}$ implies $f(T)<(a+\varepsilon)|T|$.

Proof. To show necessity assume $D_{0}(f, \alpha, x) \leqq a$ almost everywhere on $E$. Let $E_{a}=\left\{x \mid x \in E\right.$ and $\left.D_{0}(f, \alpha, x) \leqq a\right\}$. By hypothesis $\left|E_{a}\right|=|E|$. Let $\varepsilon$ be an arbitrary positive number. By Lemma 1 , for each positive integer $n$, the collection $\mathscr{F}^{n}=$ $\left\{T \mid T \in \mathscr{F}_{\alpha}\right.$, diam $(T)<1 / n$, and $\left.f(T)<(a+\varepsilon)|T|\right\}$ covers $E_{a}$ in the sense of Vitali. By the Vitali covering theorem there is a collection $\mathscr{E}_{n}$ of sets from $\mathscr{F}^{n}$ for which $\left|E_{a}-\mathscr{E}_{n}\right|<1 / n$ and hence the condition is satisfied.

To show sufficiency assume the condition holds. Let $\varepsilon>0$ be given arbitrarily. For each integer $n$, let $\mathscr{E}_{n}$ be a collection satisfying the condition. Let $B=\lim \sup \mathscr{E}_{n}$. It follows that $|E-B|=0$ since $|E-B|=\left|\lim \inf \left(E-\mathscr{E}_{n}\right)\right| \leqq \lim \inf \left|E-\mathscr{E}_{n}\right| \leqq$ $\lim \inf 1 / n=0$. But $D_{0}(f, \alpha, x) \leqq a+\varepsilon$ for any point $x \in B$ and hence almost everywhere on $E$. Since $\varepsilon$ was arbitrary, $D_{0}(f, \alpha, x) \leqq a$ almost everywhere on $E$.

Corollary 6. A necessary and sufficient condition that $D^{\circ}(f, \alpha, x) \geqq a$ almost everywhere on $E$ is obtained from Theorem 8 by replacing $D_{0}(f, \alpha, x) \leqq a$ by $D^{0}(f, \alpha, x) \geqq a$ and the condition $f(T)<(a+\varepsilon)|T|$ by $f(T)>(a-\varepsilon)|T|$.

The proof follows from Theorem 8 and Theorem 1(a).

It should be noted that in Theorem 7 the choice of $\xi$, which controls the diameter of the sets, depends upon the parameter of regularity $\alpha$. This can be seen by the following example. For any set $T$ in $\mathscr{F}$, let $f(T)=2 a|T|$ if $\operatorname{diam}(T)>r(T)$ and let $f(T)=a|T|$ if $\operatorname{diam}(T) \leqq r(T)$. Then $D^{0}(f, \alpha, x)=a$ at each point and it is evident that $\xi$ must be chosen less than $\alpha$.

There is perhaps special interest in a necessary and sufficient condition that all the $(f, \alpha)$ derivates be bounded almost everywhere on $E$. Such a condition will be 
stated as a corollary. The proof follows from Theorems 7 and 8 and Corollaries 5 and 6.

COROllary 7. Let $E$ be measurable with $|E|<+\infty$, let $\alpha$ be a real number such that $0<\alpha<1$, let $a$ be a real number and assume $\left|E-S_{\alpha}\right|=0$. A necessary and sufficient condition that both $\left|D^{0}(f, \alpha, x)\right| \leqq a$ and $\left|D_{0}(f, \alpha, x)\right| \leqq a$ is that for any $\varepsilon>0$ and $\Delta>0$, there is a number $\xi>0$ such that for any $\mathscr{E}$ satisfying $r(\mathscr{E})>\alpha$ and $\operatorname{diam}(\mathscr{E})<\xi$, the subcollection $\mathscr{E}$ at $\mathscr{E}$ consisting of all sets $T \in \mathscr{E}$ for which $|F(T)| \geqq(a+\varepsilon)|T|$ has $\left|\mathscr{E}^{\prime} \cap E\right|<\Delta$.

The final result on boundedness to be included concerns the $(\mathscr{F}, 0)$ derivate (cf. Definition 5). The domain $S_{\alpha}$ of definition of $D^{0}(f, \alpha, x)$ is such that $S_{\alpha} \subset S_{\beta}$ for $\beta<\alpha$. Let $S=\bigcup S_{\alpha}=\lim _{\alpha \rightarrow 0} S_{\alpha}$. Then $S$ is the domain of definition of $D^{0}(f, x)$.

THEOREM 9. Let $E$ be a measurable set with $|E|<+\infty$, let a be a real number, and let $|E-S|=0$. A necessary and sufficient condition that $D^{\circ}(f, x) \leqq a$ almost everywhere on $S \cap E$ is that $0<\alpha<1$ implies $D^{\circ}(f, \alpha, x) \leqq a$ almost everywhere on $S_{\alpha} \cap E$.

Proof. To prove necessity assume $D^{0}(f, x) \leqq a$ almost everywhere on $S \cap E$. Suppose the conclusion false. Since the conclusion is assumed false, there exists a value $\alpha$ for which $D^{0}(f, \alpha, x)>a$ at each point of some subset $A$ of $S_{\alpha} \cap E$ for which $|A|>0$. But $S=\bigcup_{\alpha} S_{\alpha}$ and therefore each point $x$ of $A$ is in $S$. Thus it follows from Theorem 1 (f) that $D^{0}(f, x) \geqq D^{\circ}(f, \alpha, x)$ and hence $D^{\circ}(f, x)>a$ at each point of $A$ which is a contradiction of the hypothesis.

To prove sufficiency, suppose that for each $\alpha D^{0}(f, \alpha, x) \leqq a$ almost everywhere on $E \cap S_{\alpha}$. Deny the conclusion that $D^{0}(f, x) \leqq a$ almost everywhere on $S \cap E$. Then there exists a set $A \subset S \cap E$ such that $|A|>0$ and $D^{\circ}(f, x)>a$ for each $x$ in $A$. Let $A_{\alpha}$ be the subset of $S_{\alpha} \cap E$ such that $D^{\circ}(f, \alpha, x)>a$ for each $x$ in $A_{\alpha}$. It follows from Theorem 1(f) that $A_{\alpha} \subset A_{\beta}$ for $\alpha>\beta$. Now $A=\lim _{\alpha \rightarrow 0} A_{\alpha}=\bigcup_{\alpha} A_{\alpha}$ and since $|A|>0$, there exists a value $\alpha^{\prime}$ such that $\left|A_{\alpha^{\prime}}\right|>0$. But this implies that $D^{\circ}\left(f, \alpha^{\prime}, x\right)>a$ for $x$ in $A_{\alpha^{\prime}}$ which is a contradiction of the hypothesis. Thus $D^{\circ}(f, x) \leqq a$ almost everywhere on $E \cap S_{\alpha}$.

Existence of the derivative. The previous sections have dealt with the properties of measurability, boundedness, and finiteness of the upper and lower derivates almost everywhere on certain sets. If $D^{0}\left(f, \alpha, x_{0}\right)=D_{0}\left(f, \alpha, x_{0}\right)$ then $f$ is said to be differentiable at $x_{0}$ with derivative, denoted $D\left(f, \alpha, x_{0}\right)$, equal to the common value of the derivates. The following theorem gives a necessary and sufficient condition that the derivative exist and be finite almost everywhere on a measurable set of finite measure.

TheOREM 10. Let $E$ be measurable with $|E|<+\infty$ and assume $\left|E-S_{\alpha}\right|=0$. A necessary and sufficient condition that $D(f, \alpha, x)$ exist and be finite almost everywhere 
on $E$ is that for any $\varepsilon>0$ and $\Delta>0$, there exist numbers $\eta>0$ and $\xi>0$ such that if $\mathscr{E}$ and $\mathscr{H}$ are each finite collections of disjoint sets from $\mathscr{F}$ for which $r(\mathscr{E})>\alpha$, $r(\mathscr{H})>\alpha, \operatorname{diam}(\mathscr{E})<\xi, \operatorname{diam}(\mathscr{H})<\xi,|\dot{E}-E|<\eta,|\dot{\mathscr{H}}-E|<\eta$, and $|\dot{E} \nabla \dot{\mathscr{H}}|<\eta$, then there exist subcollections $\mathscr{E}^{\prime}$ and $\mathscr{H}^{\prime}$ of $\mathscr{E}$ and $\mathscr{H}$, respectively, such that $\left|\mathscr{E}^{\prime}-\mathscr{E}^{\prime}\right|<\Delta,\left|\dot{\mathscr{H}}-\dot{\mathscr{H}}^{\prime}\right|<\Delta$, and $\left|f\left(\mathscr{E}^{\prime}\right)-f\left(\mathscr{H}^{\prime}\right)\right|<\varepsilon$.

Proof. To prove necessity, assume $D(f, \alpha, x)$ exists and is finite almost everywhere on $E$. The condition must now be shown to hold. Let $\varepsilon>0$ and $\Delta>0$ be arbitrary. It may be assumed that $\Delta \leqq|E|$ for otherwise take $\eta=(\Delta-|E|) / 2$ and all sets from each of $\mathscr{E}$ and $\mathscr{H}$ can be deleted and the condition holds. For each integer $p=1,2, \ldots$, let $E_{p}=\{x \mid x \in E$ and $|D(f, \alpha, x)|<p\}$. Let $E_{0}=\{x \mid x \in E$ and $D(f, \alpha, x)$ exists and is finite $\}$. Then $\left|E_{0}\right|=|E|, E_{p}$ is measurable for each $p$, and $\left\{E_{p}\right\}_{p=1}^{\infty}$ is an increasing sequence of sets converging upward to $E_{0}$. Let $\theta_{1}<\Delta / 4$ and let $p_{0}$ be an integer such that $|E|-\left|E_{p_{0}}\right|<\theta_{1}$. Let $\theta_{2}<\varepsilon / 32 p_{0}$ and let $G$ be an open set containing $E_{p_{0}}$ and such that $\left|G-E_{p_{0}}\right|<\theta_{2}$. Let $\theta_{3}<\min \left(\Delta / 4, \varepsilon / 32 p_{0}\right)$ and let $F$ be a closed and bounded set such that $F \subset G$ and $|G-F|<\theta_{3}$. Let $\gamma$ equal the distance between $F$ and the complement of $G$. It follows that $\gamma>0$. For any positive integers $j$ and $k$, define $E_{j}^{k}=\left\{x \mid x \in\left(F \cap E_{p_{0}}\right)\right.$ and $x \in T, r(T)>\alpha$, and $\operatorname{diam}(T)<1 / j$ implies $|f(T)|<p_{0}|T|$ and $\left.|f(T) /| T|-D(f, \alpha, x)|<1 / k\right\}$. Note that for each $j$ and $k, E_{j}^{k} \subset E_{j+1}^{k}$ and $E_{j}^{k} \supset E_{j}^{k+1}$. Furthermore, for any fixed value of $k$, $\lim _{j \rightarrow \infty} E_{j}^{k}=F \cap E_{p_{0}}$. Let $k_{0}$ be an integer such that $k_{0}>16|G| / \varepsilon$ and let $j_{0}$ be an integer such that $\left|F \cap E_{p_{0}}\right|<\left|E_{j_{0}}\right|_{e}+\theta_{3}$ [7, p. 95]. The proof will now be completed by showing that if $\eta<\min \left(\Delta / 4, \varepsilon / 16 p_{0}\right)$ and $\xi<\min \left(\gamma, 1 / j_{0}\right)$, then the condition is satisfied. Let $\mathscr{E}$ and $\mathscr{H}$ be arbitrary finite collections of sets from $\mathscr{F}$ such that $|\mathscr{E}-E|<\eta,|\dot{\mathscr{H}}-E|<\eta,|\mathscr{E} \nabla \dot{\mathscr{H}}|<\eta$, and such that $r(\mathscr{E})>\alpha, r(\mathscr{H})>\alpha, \operatorname{diam}(\mathscr{E})<\xi$, and $\operatorname{diam}(\mathscr{H})<\xi$. Delete those sets $T$ from each of the collections $\mathscr{E}$ and $\mathscr{H}$ that do not intersect $E_{j_{0}}^{k_{0}}$. Denote by $\mathscr{E}^{\prime}$ and $\mathscr{H}^{\prime}$ the remaining subcollections of $\mathscr{E}$ and $\mathscr{H}$, respectively. Notice that $\mathscr{E}^{\prime} \subset G$ and $\dot{H}^{\prime} \subset G$ because any set in $\mathscr{E}^{\prime}$ or $\mathscr{H}^{\prime}$ intersects $E_{j_{0}}^{k_{0}}$ and hence intersects $F$ and has diameter $<\xi<\gamma$, the distance from $F$ to the complement of $G$. It will now be shown that the sum of the measure of sets deleted from each of $\mathscr{E}$ and $\mathscr{H}$ is $<\Delta$.

To see that $\left|\mathscr{E}-\mathscr{E}^{\prime}\right|<\Delta$, observe that if a set $T$ was deleted it did not intersect $E_{j_{0}}^{k_{0}}$ and, therefore, $\left(\mathscr{E}-\mathscr{E}^{\prime}\right) \subset(\mathscr{E}-E) \cup\left(E-E_{p_{0}}\right) \cup\left(E_{p_{0}}-E_{j_{0}}^{k_{0}}\right)$. Furthermore,

$$
\left(E_{p_{0}}-E_{j_{0}}^{k_{0}}\right) \subset\left[(G-F) \cup\left(F-E_{j_{0}}^{k_{0}}\right)\right] \cap E_{p_{0}} \subset(G-F) \cup\left[\left(F \cap E_{p_{0}}\right)-E_{j_{0}}^{k_{0}}\right] .
$$

Thus $\left(\mathscr{E}-\mathscr{E}^{\prime}\right) \subset(\mathscr{E}-E) \cup\left(E-E_{p_{0}}\right) \cup(G-F) \cup\left[\left(F \cap E_{p_{0}}\right)-E_{j_{0}}^{k_{0}}\right]$. Let $A=\mathscr{E}-E$, $B=E-E_{p_{0}}, \quad C=G-F$, and $D=\left(\mathscr{E}-\mathscr{E}^{\prime}\right)-(A \cup B \cup C)$. Then $D \cap E_{j_{0}}^{k_{0}}=\varnothing$, $\left(D \cup E_{j 0}^{k_{0}}\right) \subset\left(F \cap E_{p_{0}}\right)$, and, since $D$ is measurable, it follows that $|D|+\left|E_{j_{0}}^{k_{0}}\right|_{e}$ $\leqq\left|F \cap E_{p_{0}}\right|$. Thus $|D|<\theta_{3}$ and

$$
\begin{aligned}
\left|\grave{E}-\mathscr{E}^{\prime}\right| & \leqq|\mathscr{E}-E|+\left|E-E_{p_{0}}\right|+|G-F|+|D| \\
& <\eta+\theta_{1}+2 \theta_{3}<\Delta / 4+\Delta / 4+\Delta / 2=\Delta .
\end{aligned}
$$

Similarly, $\left|\dot{\mathscr{H}}-\dot{\mathscr{H}}^{\prime}\right|<\Delta$. 
The proof of the necessity of the condition will now be completed by showing that $d<\varepsilon$ where $d$ is defined by $d=\left|f\left(\mathscr{E}^{\prime}\right)-f\left(\mathscr{H}^{\prime}\right)\right|$. Consider the following equivalent form for $d$ :

$$
d=\left|\sum_{S \in \mathscr{\mathscr { S }}^{\prime}}[f(S) /|S|] \cdot\right| S\left|-\sum_{T \in \mathscr{H}^{\prime}}[f(T) /|T|] \cdot\right| T|| .
$$

The value of $d$ can be calculated by $d=\left|\int_{\mathscr{E}^{\prime}} g(x) d x-\int_{\mathscr{\mathscr { C }}^{\prime}} h(x) d x\right|$ where the integrals are Lebesgue integrals and where the real valued point functions $g(x)$ and $h(x)$ are defined as

$$
\begin{aligned}
& g(x)=f(S) /|S| \text { if } x \in S \text { for some } S \in \mathscr{E}^{\prime} \text { and } g(x)=0 \text { otherwise, and } \\
& h(x)=f(T) /|T| \text { if } x \in T \text { for some } T \in \mathscr{H}^{\prime} \text { and } h(x)=0 \text { otherwise. }
\end{aligned}
$$

Each of the point functions $g(x)$ and $h(x)$ are single valued and can take only a finite number of values. Furthermore, since each set in $\mathscr{E}^{\prime}$ or in $\mathscr{H}^{\prime}$ intersects $E_{j_{0}}^{k_{0}}$, it follows from the definition of $E_{j_{0}}^{k_{0}}$ that $|g(x)|<p_{0}$ and $|h(x)|<p_{0}$. Let $A=\mathscr{E}^{\prime \prime} \cap \dot{\mathscr{H}}^{\prime}$. Then $d$ can be written in the form

$$
d=\left|\int_{A}[g(x)-h(x)] d x+\int_{\dot{\mathscr{O}}^{\prime}-A} g(x) d x-\int_{\dot{\mathscr{H}}^{\prime}-A} h(x) d x\right| .
$$

Notice that $A$ is the union of a finite number of disjoint sets, each of which has the form $S \cap T$ for some $S \in \mathscr{E}^{\prime}$ and $T \in \mathscr{H}^{\prime}$. Some of these sets may intersect $E_{j_{0}}^{k_{0}}$ and others may not. Let $A=A_{1} \cup A_{2}$ where $A_{1}$ is the union of those sets which intersect $E_{j_{0}}^{k_{0}}$ and $A_{2}$ is the union of those sets which do not intersect $E_{j_{0}}^{k_{0}}$. Using the triangle inequality, $d$ may be written in the form

$$
\begin{aligned}
d \leqq & \left|\int_{A_{1}}[g(x)-h(x)] d x\right|+\left|\int_{A_{2}}[g(x)-h(x)] d x\right| \\
& +\left|\int_{\dot{\delta}^{\prime}-A} g(x) d x\right|+\left|\int_{\dot{\mathscr{H}}^{\prime}-A} h(x) d x\right| .
\end{aligned}
$$

A bound will now be found for each of these four integrals. Notice that $x \in A_{1}$ implies that both $g(x)$ and $h(x)$ are within $1 / k_{0}$ of $D(f, \alpha, x)$ and, therefore, $|g(x)-h(x)|<2 / k_{0}$. Therefore the first integral in (1) satisfies the inequality

(2) $\left|\int_{A_{1}}[g(x)-h(x)] d x\right| \leqq \int_{A_{1}}|g(x)-h(x)| d x \leqq\left(2 / k_{0}\right)\left|A_{1}\right| \leqq\left(2 / k_{0}\right)|G|<\varepsilon / 4$.

In order to establish an upper bound for the second integral in (1), a bound is needed for $\left|A_{2}\right|$. Since $A_{2} \cap E_{j_{0}}^{k_{0}}=\varnothing$ and $E_{j_{0}}^{k_{0}} \subset F \cap E_{p_{0}}$, it follows that

$$
\left|E_{j_{0}}^{k_{0}}\right|_{e} \leqq\left|F \cap E_{p_{0}}\right|-\left|A_{2} \cap F \cap E_{p_{0}}\right|
$$

Thus

$$
\left|A_{2} \cap F \cap E_{p_{0}}\right| \leqq\left|F \cap E_{p_{0}}\right|-\left|E_{j_{0}}^{k_{0}}\right|_{e}<\theta_{3} .
$$

Also since $A_{2} \subset G$, it follows that

$$
\left|A_{2}\right| \leqq\left|A_{2} \cap F \cap E_{p_{0}}\right|+\left|G-E_{p_{0}}\right|+|G-F|<\theta_{2}+2 \theta_{3} .
$$


Now since $|g(x)-h(x)| \leqq|g(x)|+|h(x)|<2 p_{0}$, the second integral of (1) satisfies the inequality

$$
\left|\int_{A_{2}}[g(x)-h(x)] d x\right| \leqq \int_{A_{2}}|g(x)-h(x)| d x \leqq 2 p_{0}\left|A_{2}\right|<2 p_{0}\left(\theta_{2}+2 \theta_{3}\right)
$$

The last two integrals of (1) will be considered together.

(4) $\left|\int_{\dot{\mathscr{E}}^{\prime}-A} g(x) d x\right|+\left|\int_{\dot{\mathscr{H}}^{\prime}-A} h(x) d x\right|<p_{0}\left[\left|\mathscr{E}^{\prime}-A\right|+\left|\dot{\mathscr{H}}^{\prime}-A\right|\right]=p_{0}\left|\mathscr{E}^{\prime} \nabla \dot{\mathscr{H}}^{\prime}\right|$.

Therefore, an upper bound is needed for the symmetric difference of $\mathscr{E}^{\prime}$ and $\dot{\mathscr{H}}^{\prime}$. Since $\mathscr{E}^{\prime} \subset G$,

$$
\begin{aligned}
\mathscr{E}^{\prime}-\dot{\mathscr{H}}^{\prime} & \subset\left[\left(G \cap \mathscr{E}^{\prime}\right)-\dot{\mathscr{H}}^{\prime}\right] \subset\left[\left(G \cap \dot{\mathscr{E}}^{\prime}\right)-E_{p_{0}}\right] \cup\left[\left(E_{p_{0}} \cap \mathscr{E}^{\prime}\right)-\dot{\mathscr{H}}^{\prime}\right] \\
& \subset\left(G-E_{p_{0}}\right) \cup\left[\left(E_{p_{0}} \cap \mathscr{E}^{\prime}\right)-\left(\dot{\mathscr{H}} \cap E_{p_{0}}\right)\right] \cup\left[\left(\mathscr{\mathscr { H }} \cap E_{p_{0}}\right)-\dot{\mathscr{H}}^{\prime}\right] .
\end{aligned}
$$

Now $\left[\left(E_{p_{0}} \cap \mathscr{E}^{\prime}\right)-\left(\dot{\mathscr{H}} \cap E_{p_{0}}\right)\right] \subset\left(\mathscr{E}^{\prime}-\dot{\mathscr{H}}\right) \subset(\mathscr{E}-\dot{\mathscr{H}}) \subset(\mathscr{E} \nabla \dot{\mathscr{H}})$. If $x \in \dot{\mathscr{H}}$ but $x \notin \dot{\mathscr{H}}^{\prime}$, then $x$ was in a set $T$ which was deleted from $\mathscr{H}$ and hence $x \notin E_{j_{0}}^{k_{0}}$. Therefore,

$$
\begin{aligned}
& {\left[\left(\dot{\mathscr{H}} \cap E_{p_{0}}\right)-\dot{\mathscr{H}}^{\prime}\right] \subset[(}\left.\left.\dot{\mathscr{H}} \cap E_{p_{0}}\right)-E_{j_{0}}^{k_{0}}\right] \subset\left[\left(\dot{\mathscr{H}} \cap E_{p_{0}}\right)-\left(F \cap E_{p_{0}}\right)\right] \\
& \cup\left[\left(F \cap E_{p_{0}}\right)-E_{j_{0}}^{k_{0}}\right] \subset(G-F) \cup\left[\left(F \cap E_{p_{0}}\right)-E_{j_{0}}^{k_{0}}\right]
\end{aligned}
$$

and hence

$$
\mathscr{E}^{\prime}-\dot{\mathscr{H}}^{\prime} \subset\left(G-E_{p_{0}}\right) \cup(\mathscr{E} \nabla \dot{\mathscr{H}}) \cup(G-F) \cup\left[\left(F \cap E_{p_{0}}\right)-E_{j_{0}}^{k_{0}}\right] .
$$

Let $D=\left(\mathscr{E}^{\prime}-\dot{\mathscr{H}}^{\prime}\right)-\left[\left(G-E_{p_{0}}\right) \cup(\mathscr{E} \nabla \dot{\mathscr{H}}) \cup(G-F)\right]$. Then $D \cap E_{j_{0}}^{k_{0}}=\varnothing, D \cup E_{j_{0}}^{k_{0}}$ $\subset F \cap E_{p_{0}}$, and, since $D$ is measurable, it follows that

$$
|D| \leqq\left|F \cap E_{p_{0}}\right|-\left|E_{j_{0}}^{k_{0}}\right|_{e}<\theta_{3} .
$$

Thus, $\left|\mathscr{E}^{\prime}-\dot{\mathscr{H}}^{\prime}\right| \leqq \theta_{2}+\eta+2 \theta_{3}$. Similarly, $\left|\dot{\mathscr{H}}^{\prime}-\mathscr{E}^{\prime}\right| \leqq \theta_{2}+\eta+2 \theta_{3}$ and hence

$$
\left|\mathscr{E}^{\prime} \nabla \dot{\mathscr{H}}^{\prime}\right|<2\left(\theta_{2}+\eta+2 \theta_{3}\right) \text {. }
$$

Therefore, using (2), (3), (4), and (5), the value of $d$ expressed in (1) satisfies the inequality

$$
d \leqq \varepsilon / 4+2 p_{0}\left(\theta_{2}+2 \theta_{3}\right)+2 p_{0}\left(\theta_{2}+\eta+2 \theta_{3}\right)<\varepsilon .
$$

This concludes the proof of the necessity of the condition.

To prove sufficiency, assume the condition holds. Suppose the theorem false. Then either $D^{0}(f, \alpha, x)=+\infty$ or $D_{0}(f, \alpha, x)=-\infty$ on a subset $A$ of $E$ with $|A|>0$ or else there exist rational numbers $\beta, \gamma, \lambda, \nu$ such that the set $A=\{x \mid x \in E$ and $\left.\beta>D^{0}(f, \alpha, x)>\gamma>\lambda>D_{0}(f, \alpha, x)>\nu\right\}$ has $|A|>0$.

Suppose the latter case. Let $\varepsilon=(\gamma-\lambda)|A| / 2$ and let $\Delta=(\gamma-\lambda)|A| / 4(|\beta|+|\nu|)$. It will now be shown that the condition of the theorem fails to hold for these values of $\varepsilon$ and $\Delta$. Let $\eta>0$ and $\xi>0$ be arbitrary. Let

$$
\theta=\min (\eta / 4,|A| / 2,(\gamma-\lambda)|A| / 4(|\gamma|+|\lambda|)) .
$$


Let $G$ be an open set such that $A \subset G$ and $|G-A|<\theta$. Using Lemma 1, it can be shown that the collection $\mathscr{F}^{\prime}=\left\{T \mid T \in \mathscr{F}_{\alpha}, T \subset G\right.$, diam $(T)<\xi$, and $\left.\beta>f(T) /|T|>\gamma\right\}$ covers $A$ in the sense of Vitali. By the Vitali covering theorem let $\mathscr{E}$ be a finite collection from $\mathscr{F}^{\prime}$ such that $|A-\mathscr{E}|<\theta$. Similarly, $\mathscr{F}^{\prime \prime}=\left\{T \mid T \in \mathscr{F}_{\alpha}, T \subset G, \operatorname{diam}(T)\right.$ $<\xi$, and $\lambda>f(T) /|T|>\nu\}$ covers $A$ in the sense of Vitali. Let $\mathscr{H}$ be a collection from $\mathscr{F}^{\prime \prime}$ such that $|A-\dot{\mathscr{H}}|<\theta$. Now $A \nabla \mathscr{E} \subset(A-\mathscr{E}) \cup(G-A)$ and, therefore, $|A \nabla \mathscr{E}|$ $<2 \theta$. Similarly, $|A \nabla \dot{\mathscr{H}}|<2 \theta$. Since $(\mathscr{E} \nabla \dot{\mathscr{H}}) \subset(\mathscr{E} \nabla A) \cup(\dot{\mathscr{H}} \nabla A)$, it follows that $|\mathscr{E} \nabla \dot{\mathscr{H}}|<4 \theta<\eta$. Since $\mathscr{E} \subset G$ and $\dot{\mathscr{H}} \subset G$, then $|\mathscr{E}-E| \leqq|G-E| \leqq|G-A|<\eta$ and, similarly, $|\dot{\mathscr{H}}-E|<\eta$. Let $\mathscr{E}^{\prime}$ and $\mathscr{H}^{\prime}$ be arbitrary subcollections of $\mathscr{E}$ and $\mathscr{H}$, respectively, such that $\left|\mathscr{E}-\mathscr{E}^{\prime}\right|<\Delta$ and $\left|\dot{H}^{\prime}-\dot{\mathscr{H}}^{\prime}\right|<\Delta$. Now $f\left(\mathscr{E}^{\prime}\right)-f\left(\mathscr{H}^{\prime}\right)=f(\mathscr{E})$ $-f\left(\mathscr{E}-\mathscr{E}^{\prime}\right)-f(\mathscr{H})+f\left(\mathscr{H}-\mathscr{H}^{\prime}\right)$. Also, $T \in \mathscr{E}$ implies $\gamma|T|<f(T)<\beta|T|$ and $T \in \mathscr{H}$ implies $\nu|T|<f(T)<\lambda|T|$. Using these inequalities, it follows that

$$
\begin{aligned}
f\left(\mathscr{E}^{\prime}\right)-f\left(\mathscr{H}^{\prime}\right) & \geqq f(\mathscr{E})-f(\mathscr{H})-|B|\left|\mathscr{E}^{\prime}-\mathscr{E}^{\prime}\right|-|\nu|\left|\dot{\mathscr{H}}^{\prime}-\dot{\mathscr{H}}^{\prime}\right| \\
& \geqq \gamma|\mathscr{E}|-\lambda|\dot{\mathscr{H}}|-(|\beta|+|\nu|) \Delta .
\end{aligned}
$$

By adding and subtracting $(\gamma+\lambda)|A|$ this inequality can be written

$$
f\left(\mathscr{E}^{\prime}\right)-f\left(\mathscr{H}^{\prime}\right) \geqq(\gamma-\lambda)|A|-|\gamma|[|| \mathscr{E}|-| A||]-|\lambda|[|| A|-| \dot{\mathscr{H}}||]-(|\beta|+|\nu|) \Delta .
$$

Since $|A|-|\mathscr{E}| \leqq|A-\mathscr{E}|<\theta$ and $|\mathscr{E}|-|A| \leqq|\mathscr{E}-A|<|G-A|<\theta$, it follows that || $\mathscr{E}|-| A||<\theta$. Similarly, ||$\dot{\mathscr{H}}|-| A||<\theta$. Consequently,

$$
f\left(\mathscr{E}^{\prime}\right)-f\left(\mathscr{H}^{\prime}\right) \geqq(\gamma-\lambda)|A|-(|\gamma|+|\lambda|) \theta-(|\beta|+|\nu|) \Delta .
$$

Substituting the appropriate values for $\theta$ and $\Delta$, the above inequality can be written in the form

$$
f\left(\mathscr{E}^{\prime}\right)-f\left(\mathscr{H}^{\prime}\right) \geqq(\gamma-\lambda)|A|-(\gamma-\lambda)|A| / 4-(\gamma-\lambda)|A| / 4=\varepsilon .
$$

Thus, since $f\left(\mathscr{E}^{\prime}\right)-f\left(\mathscr{H}^{\prime}\right)>0$, it follows that $\left|f\left(\mathscr{E}^{\prime}\right)-f\left(\mathscr{H}^{\prime}\right)\right|=f\left(\mathscr{E}^{\prime}\right)-f\left(\mathscr{H}^{\prime}\right) \geqq \varepsilon$. This is a contradiction of the hypothesis that the condition holds and results from the supposition that $\beta>D^{0}(f, \alpha, x)>\gamma>\lambda>D_{0}(f, \alpha, x)>\nu$ for values of $x$ in a set $A$ of positive measure.

Suppose now the other alternative, that $D^{\circ}(f, \alpha, x)=+\infty$ or $D_{0}(f, \alpha, x)=-\infty$ on a subset $A$ of $E$ with $|A|>0$. If $D^{\circ}(f, \alpha, x)=+\infty$ at each point $x$ of a subset $A$ of $E$ with $|A|>0$, let $\varepsilon=|A| / 4$ and let $\Delta=|A| / 4$. It will now be shown that for these values of $\varepsilon$ and $\Delta$ the condition of the theorem will not hold. Let $\eta$ and $\xi$ be positive numbers. Let $0<\theta<\min (\Delta, \eta / 4)$ and let $G$ be an open set such that $A \subset G$ and $|G-A|<\theta$. By Lemma 1, the collection $\mathscr{F}^{\prime}=\left\{T \mid T \in \mathscr{F}_{\alpha}, T \subset G\right.$, diam $(T)<\xi$, and $f(T) /|T|>0\}$ covers $A$ in the sense of Vitali. By the Vitali covering theorem let $\mathscr{E}$ be a collection of sets from $\mathscr{F}^{\prime}$ such that $|A-\mathscr{E}|<\theta$. Since there are only a finite number of sets in $\mathscr{E}$, let $a=\max _{T \in \mathscr{E}} f(T) /|T|$. Also, by Lemma 1 , the collection $\mathscr{F}^{\prime \prime}=\left\{T \mid T \in \mathscr{F}_{\alpha}, T \subset G, \operatorname{diam}(T)<\xi\right.$, and $\left.f(T) /|T|>(5 a / 2+1)\right\}$ covers $A$ in the sense of Vitali. By the Vitali covering theorem let $\mathscr{H}$ be a collection from $\mathscr{F}^{\prime \prime}$ such that $|A-\dot{\mathscr{H}}|<\theta$. Observe that $|\mathscr{E}-E| \leqq|\mathscr{E}-A| \leqq|G-A|<\eta$ and, similarly, $|\dot{\mathscr{H}}-E|<\eta$. 
Also, $|\mathscr{E} \nabla \dot{\mathscr{H}}| \leqq|\mathscr{E} \nabla A|+|\dot{\mathscr{H}} \nabla A|<\eta$. Now $|\dot{\mathscr{H}}| \geqq|A \cap \dot{\mathscr{H}}| \geqq|A|-|A-\dot{\mathscr{H}}|>$ $|A|-\theta$. Let $\mathscr{E}^{\prime}$ and $\mathscr{H}^{\prime}$ be any subcollections of $\mathscr{E}$ and $\mathscr{H}$, respectively, such that $\left|\mathscr{E}^{\prime}-\mathscr{E}^{\prime}\right|<\Delta$ and $\left|\dot{\mathscr{H}}-\dot{\mathscr{H}}^{\prime}\right|<\Delta$. Then

and

$$
\left|\mathscr{E}^{\prime}\right| \leqq|G|<|A|+\theta \leqq 5|A| / 4
$$

Then

$$
\left|\dot{\mathscr{H}}^{\prime}\right|>|\dot{\mathscr{H}}|-\Delta>|A|-\theta-\Delta>|A|-2 \theta>|A| / 2
$$

$$
f\left(\mathscr{E}^{\prime}\right) \leqq a\left|\mathscr{\mathscr { E }}^{\prime}\right|<5 a|A| / 4 \text { and } f\left(\mathscr{H}^{\prime}\right) \geqq(5 a / 2+1)\left|\dot{\mathscr{H}}^{\prime}\right|>(5 a / 2+1)|A| / 2 .
$$

Therefore,

$$
\left|f\left(\mathscr{H}^{\prime}\right)-f\left(\mathscr{E}^{\prime}\right)\right|=f\left(\mathscr{H}^{\prime}\right)-f\left(\mathscr{E}^{\prime}\right)>5 a|A| / 4+|A| / 2-5 a|A| / 4=|A| / 2>\varepsilon
$$

which is a contradiction that the condition holds. Similarly, if $D_{0}(f, \alpha, x)=-\infty$ on a set $A$ with $|A|>0$, it can be shown that the condition of the theorem does not hold. This completes the proof of Theorem 10.

It is noted that the hypothesis $|E|<+\infty$ is necessary in Theorem 10. This can be seen by the following example. Let the space be the Euclidean plane, $R_{2}$, and let $E=R_{2}$. Let the family $\mathscr{F}$ of sets consist of all circular discs in the plane with diameter $<1$. For each integer $n \geqq 0$, let $C_{n}$ denote the closed circular disc with center at the origin and with radius $n$. For each integer $n \geqq 1$, let $A_{n}=C_{n}-C_{n-1}$. Define the function $f$ as follows: If $T$ is a circular disc with center at the origin, let $f(T)=|T|$, but if $T$ is a circular disc with center in $A_{n}$, let $f(T)=|T|$ if $\operatorname{diam}(T)$ $<1 / n$ and let $f(T)=2|T|$ if $\operatorname{diam}(T) \geqq 1 / n$. Clearly $D(f, \alpha, x)=1$ at every point in $E$. To see that the condition of Theorem 10 fails to hold, let $\varepsilon>0$ and $\Delta>0$ be given. It suffices to show that for any $\eta>0$ and $\xi>0$, collections $\mathscr{E}$ and $\mathscr{H}$ can be chosen so that the condition fails. Let $\eta>0$ and $\xi>0$ be arbitrary. Let $N$ be an integer which is sufficiently large that a collection $\mathscr{E}$ can be chosen with the properties that $|\mathscr{E}|>\varepsilon+2 \Delta+\eta$ and that $T \in \mathscr{E}$ implies $1 / N<\operatorname{diam}(T)<\xi$ and $T \subset A_{N}^{0}$, where $A_{N}^{0}$ denotes the interior of $A_{N}$. The existence of such an integer $N$ follows from the fact that $\lim _{n \rightarrow \infty}\left|A_{n}\right|=+\infty$. Let $G$ be an open set such that $G \subset A_{N}^{0}$, $\mathscr{E} \subset G$ and $|G-\mathscr{E}|<\eta / 2$. Now the collection $\mathscr{F}^{\prime}=\{T \mid T \in \mathscr{F}, T \subset G$ and $\operatorname{diam}(T)$ $<1 / N\}$ covers $\mathscr{E}$ in the sense of Vitali. By the Vitali covering theorem let $\mathscr{H}$ be a collection from $\mathscr{F}^{\prime}$ such that $|\mathscr{E}-\dot{\mathscr{H}}|<\eta / 2$. Then $|\mathscr{E} \nabla \dot{\mathscr{H}}| \leqq|\mathscr{E}-\dot{\mathscr{H}}|+|G-\mathscr{E}|<\eta$. Let $\mathscr{E}^{\prime}$ and $\mathscr{H}^{\prime}$ be subcollections of $\mathscr{E}$ and $\mathscr{H}$, respectively, such that $\left|\mathscr{E}-\mathscr{E}^{\prime}\right|<\Delta$ and $\left|\dot{\mathscr{H}}-\dot{\mathscr{H}}^{\prime}\right|<\Delta$. Thus $f\left(\mathscr{E}^{\prime}\right)=2\left|\mathscr{E}^{\prime}\right|$ and $f\left(\mathscr{H}^{\prime}\right)=\left|\dot{\mathscr{H}}^{\prime}\right|$ and, since $|\dot{\mathscr{H}}|-\left|\mathscr{E}^{\prime}\right|$ $\leqq|\dot{\mathscr{H}}-\mathscr{E}| \leqq|G-\mathscr{E}|<\eta,|\dot{\mathscr{H}}| \leqq|\mathscr{E}|+\eta$. Consequently,

$$
f\left(\mathscr{E}^{\prime}\right)-f\left(\mathscr{H}^{\prime}\right)=2\left|\mathscr{E}^{\prime}\right|-\left|\dot{\mathscr{H}}^{\prime}\right| \geqq 2\left(\left|\mathscr{E}^{\prime}\right|-\Delta\right)-|\dot{\mathscr{H}}| \geqq 2\left|\mathscr{E}^{\prime}\right|-2 \Delta-|\mathscr{E}|-\eta>\varepsilon .
$$

Thus the condition of Theorem 10 fails to hold.

The final theorem concerns the existence of the $(\mathscr{F}, 0)$ derivative (cf. Definition 5 ).

THEOREM 11. Let $E$ be a measurable set of finite measure, and let $|E-S|=0$ (see Theorem 9). A necessary and sufficient condition that $D(f, x)$ exists and is finite 
almost everywhere on $S \cap E$ is that $0<\alpha<1$ implies $D(f, \alpha, x)$ exists and is finite almost everywhere on $S_{\alpha} \cap E$.

Proof. It was pointed out in connection with Theorem 9 that $S_{\alpha} \subset S_{\beta}$ for $\alpha>\beta$ and also that $S=\bigcup_{\alpha} S_{\alpha}=\lim _{\alpha \rightarrow 0} S_{\alpha}$. From Theorem 1(f) it is seen that $D^{0}(f, \alpha, x)$ is nondecreasing and $D_{0}(f, \alpha, x)$ is nonincreasing as $\alpha \rightarrow 0$. Therefore, it follows that: (1) if $x \in S_{\alpha}$ and $D^{\circ}(f, \alpha, x)=+\infty$ then $D^{0}(f, x)=+\infty$, (2) if $x \in S_{\alpha}$ and $D_{0}(f, \alpha, x)=-\infty$ then $D_{0}(f, x)=-\infty$, or (3) if $x \in S_{\alpha}$ and $D^{0}(f, \alpha, x)>D_{0}(f, \alpha, x)$ then $D^{0}(f, x)>D_{0}(f, x)$. Consequently, if $A_{\alpha}$ is the subset of $S_{\alpha}$ on which $D(f, \alpha, x)$ either does not exist or is infinite, then $A_{\alpha} \subset A_{\beta}$ for $\alpha>\beta$ and if $A$ is the subset of $S$ for which $D(f, x)$ does not exist or is infinite, then $A=\bigcup_{\alpha} A_{\alpha}=\lim _{\alpha \rightarrow 0} A_{\alpha}$. Also, if $D(f, x)$ exists and is finite at some point $x \in S_{\alpha}$, then $D(f, \alpha, x)$ exists and is finite and, furthermore, $\beta<\alpha$ implies $D(f, \beta, x)$ exists and is finite and $D(f, \alpha, x)=$ $D(f, \beta, x)=D(f, x)$. The proof of the theorem follows easily from these remarks. To prove the sufficiency, assume that, for $0<\alpha<1, D(f, \alpha, x)$ exists and is finite almost everywhere on $S_{\alpha} \cap E$. Then $\left|A_{\alpha} \cap E\right|=0$ for each $\alpha$ and since $A=\lim _{\alpha \rightarrow 0} A_{\alpha}$, it follows that $|A \cap E|=\lim _{\alpha \rightarrow 0}\left|A_{\alpha} \cap E\right|=0$. To prove necessity, assume $D(f, x)$ exists and is finite almost everywhere on $S \cap E$. Then $|A \cap E|=0$ and, since $A_{\alpha} \subset A,\left|A_{\alpha} \cap E\right|=0$.

\section{BIBLIOGRAPHY}

1. S. Banach, Sur une classe de fonctions d'ensemble, Fund. Math. 6 (1924), 170-188.

2. Casper Goffman, Real functions, Rinehart, New York, 1953.

3. Hans Hahn and Arthur Rosenthal, Set functions, Univ. of New Mexico Press, Albuquerque, New Mexico, 1948.

4. W. E. Hartnett and A. H. Kruse, Differentiation of set functions using Vitali coverings, Trans. Amer. Math. Soc. 96 (1960), 185-209.

5. Henri Lebesgue, Sur l'intégration des fonctions discontinues, Ann. Sci. École Norm. Sup. (3) 27 (1910), 361-450.

6. Edward J. McShane, Integration, Princeton Univ. Press, Princeton, N. J., 1947.

7. M. E. Munroe, Introduction of measure and integration, Addison-Wesley, Reading, Mass., 1953.

8. Anthony P. Morse, A theory of covering and differentiation, Trans. Amer. Math. Soc. 55 (1944), 205-235.

9. C. Y. Pauc, Review \#12195, Math. Reviews 22 (1961), p. 2085.

10. René de Possel, Sur la derivation abstraite des fonctions d'ensembles, C. R. Acad. Sci. Paris 201 (1935), 579.

11. S. Saks, Théorie de l'intégrale, Monografie Matematyczne, vol. II, PWN, Warsaw, 1933; English transl., Monografie Matematyczne, vol. VII, PWN, Warsaw, 1937; 2nd rev. ed., Dover, New York, 1964.

12. A. J. Ward, On the derivation of additive functions of intervals, Fund. Math. 28 (1937), 265-279.

13. R. C. Young, Functions of $\sum$ defined by addition or functions of intervals in n-dimensional formulation, Math. Z. 29 (1928), 171-216.

OAK Ridge National Laboratory, OAK Ridge, TENNESSEE 